\title{
Studio preliminare sulle oscillazioni libere del Lago di Sauris a due diverse quote (*)
}

\author{
G. Romualdi - M. C. Spadea \\ Ricevuto il 30 Maggio 1966
}

Riassunto. -.- In questa prima parte, viene applicato al Lago di Sauris il metodo di Hidaka — opportunamente esteso - allo scopo di stu. diarne le oscillazioni libere.

La ricerca è stata particolarmente interessante per $i$ due seguenti motivi:

1. - Si tratta di un bacino artificiale formato dallo sbarramento del torrente Lumiei (Diga del Lumiei alta $130 \mathrm{~m}$ ).

2. - Si è potuto studiare il Lago a due diverse quote $(980 \mathrm{~m}$, invaso pieno - e $939 \mathrm{~m}$, quota allegerimento), fatto che è pressoché impossibile riscontrare in un lago naturale.

Sono stati cosi determinati i periodi, la posizione dei nodi e le ampiezze corrispondenti alla sessa uni- e bi-nodale alle due diverse quote.

Nella seconda parte, di prossima pubblicazione, lo studio sarà esteso al confronto fra i dati qui ottenuti per via teorica e quelli ottenuti per via sperimentale mediante registrazioni limnografiche.

Summary. - In the first stage concerning the free oscillations of the Sauris' Lake, we limited ourselves to the periods, knots and amplitudes determination of the uni and bi-nodal seiche, making use of the Hidaka's method. The investigation is particularly interesting due to two particular reasons:

1. - It is an artificial reservoir, formed barring the Lumiei torrent. (Lumiei Dam, high $130 \mathrm{~m}$ ).

2. - The Lake has been studied at two different altitudes (whole basin, $980 \mathrm{~m}$ - and $939 \mathrm{~m}$ ), matter which in a natural lake is almost impossible to find.

In the second part, the research will be exended to the comparison of the theoretical results. here achieved-with the experimental results obtained by limnographic records.

(*) Questo lavoro ò stato compiuto con contributi del C.I.R. 


\section{PARTE I}

\section{Premessa.}

L'accordo fra teoria (applicazione di vari metodi matematici di Chrystal, Defant, Пidaka, Goldberg, alcuni dei quali opportunamente estesi) e osservazione (registrazioni ottenute con limnografi nello studio delle oscillazioni libere di laghi naturali, golfi, etc...) ì stato più volte confermato, come risulta dai numerosi lavori già pubblicati.

Il lavoro è stato diviso in due parti. Nella prima, che concerne questa nota, il lago artificiale di Sauris viene studiato dal punto di vista teorico; nella seconda parte - di prossima pubblicazione - lo scopo sarà quello di dimostrare che l'aceordo sopra menzionato viene rispettato, anche nel aso di laghi artificiali quale è quello del Lumiei, formatosi quest'ultimo dopo la costruzione della diga sul torrente omonimo, uno dei tanti affuenti del Tagliamento.

1. - La diga del Lumiei è sita in Iocalità Maina di Sauris (Carnia) a $1000 \mathrm{~m}$. di altezza s.m., del tipo ad arco-cupola (areo a doppia curvatura); essa è alta $136 \mathrm{~m}$, con uno spessore di $16 \mathrm{~m}$ alla base e di $3,5 \mathrm{~m}$ al coronamento (sezione maestra), ed ha (reato un serbatoio della capacità utile di 70 milioni di $\mathrm{m}^{3}$.

Il lago di Sauris dopo un'ansa piuttosto larga a monte della diga, si restringe notevolmente assumendo una forma molto allungata. La profondita massima si ha appunto in corrispondenza di questa ansa, ed essa decresce rapidamente in concomitanza al diminuire della larghezza, tanto da essere minima all'estremo opposto.

Nel caso specifico vanno sottolineati tre aspetti del lago:

a) è un bacino artificiale;

b) di forma irregolare (vedi curva normale: Figer. 1-2);

c) è soggetto a forti variazioni di livello.

Quest'ultima caratteristica ci ha permesso di studiare il lago di Sauris in due diverse situazioni di invaso: a invaso pieno $(\mathrm{m} .980) \mathrm{e}$ ad una quota di alleggerimento (m 939), quasi si trattasse di due laghi diversi, tali risultando dal punto di vista morfometrico.

A nostro avviso il punto c) è il più significativo, dato che non è possibile usufruire in natura di un lago che, in epoche diverse, presenti differenze di quota di oltre 40 metri. 
Ta prima parte di questa nota si riferisee appunto allo studio delle oscilbazioni libere del lagu di Sauris a invaso pieno e a quota di alleggorimento (41 metri sotto il livello normale).

Va ancora messo in evidenza che la batimetria del lago è perfettamente conosciuta, a chusa del rigoroso rilevamento topografico cui il bacino è stato sottoposto prima della costruzione delli diga; ciò che orviamente non avviene per i laghi naturali la cui batimetria è sovente inesatta, in ogni cuso otienuta con un troppo limitato numero di sondaggi, ripetuti soltanto a lunghi intervalli di tempo.

LAGO DI SAZRIS A QUOTA 980.

2. - I principali elementi morfometrici del lago di Sauris a bacino pieno sono:

$\begin{array}{ll}\text { altezza } & \mathrm{m} .980 \mathrm{s.m} . \\ \text { superficie } & \mathrm{km}^{2} 1.765 .122 \\ \text { profondità missima } & \mathrm{m} 140 .\end{array}$

3. - Dato il particolare tipo di lago, abbiamo ritenuto opportuno applicare il metodo di Hidaka (2), il più idoneo per laghi irregolari, la cui curva normale risulti complicata (Fig. 2).

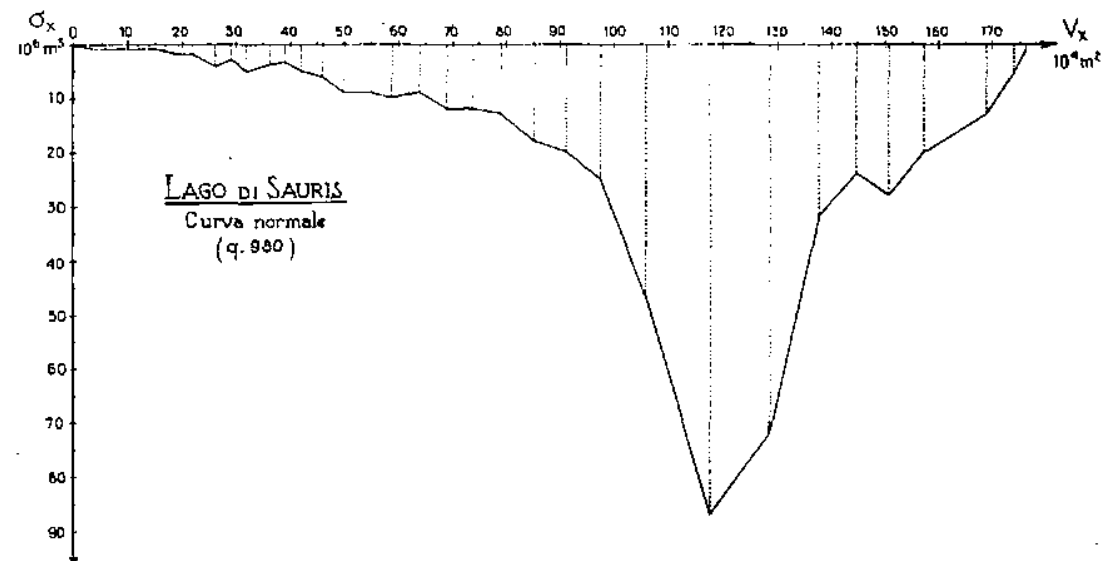

Fig. 2

Nel caso in esame per ottenere i dati necessari alla determinazione di detta curva ci siamo serviti di una carta batimetrica del lago 1 : 2000 (disegno fornito a suo tempo dalla SADE). 
L'estension. 1.] metodo $\left({ }^{3}\right)$, non solo consente di determinare $i$ periodi delle oscillazioni libere, ma anche la posizione delle linee nodali, nonché l'ampix'zza de'ry spostimenti verticali nelle singole sezioni trasversali, traceiate perpendicolarmente alla linea di valle dall'estremo 0 alla diga; dette sezono distano una dallable $100 \mathrm{~m}$ (Fig. 3). Tutti i dati numerici neeessarei all'applieazione del metodo sono riportati nella Tabella I.

4. - Metodo di Hidaka. Senza solüermarsi sulla teoria, già più volte ampiamente esposta : opportunamente ampliata $\left({ }^{3}\right)$, richiamiamo solo le formule fondamentali, neessaric a formire i dati numerici per il nostro lavoro.

Detto $\xi$ lo spostamento orizzontale delle particelle liquide di una stessa sezione trusversal $\left(\cdot S(x)\right.$ e posto $u=\xi \cdot S(x) ; v-\int_{0}^{x} b(x) d x$, dove $b(x)$ rappresenta la larenheza variabile del bacino misurata alla superficie libera, l'equazione sul moto libero dei laghi (equazione di Chrystal) (1) assume la forma:

$$
\sigma(v) \frac{d^{2} u}{d v^{2}}+\frac{4 \pi^{2}}{g T^{2}} u=0
$$

In essa, valgono le condizioni ai limiti $u(o)=u(a)=0$ (a $=\mathrm{su}$ perficie totale del lagro).

Nella [1] $\sigma(v)=S^{\prime}(x) \cdot b(x)$ rappresenta la curva normale del lago (con $\sigma(v)$ che si arnulia agli estremi in modo che sia $\sigma(o)=\sigma(a)=0$ ) nella quale i valori di $\sigma(v)$ sono riportati in ordinata e quelli del$l^{\prime} \int b(x) d x$ in ascissa.

$$
\begin{aligned}
& \text { Posto } z=\frac{v}{a} \text { la [1] diviene: } \\
& \qquad \begin{array}{l}
\frac{d^{2} u}{d z^{2}}+\frac{\dot{\lambda}}{\sigma(z)} u=0,
\end{array}
\end{aligned}
$$

con le condizioni ai limiti

$$
u(0)=u(1)=0
$$

e

$$
\lambda=\frac{4 \pi^{2} a^{2}}{g T^{2}}
$$




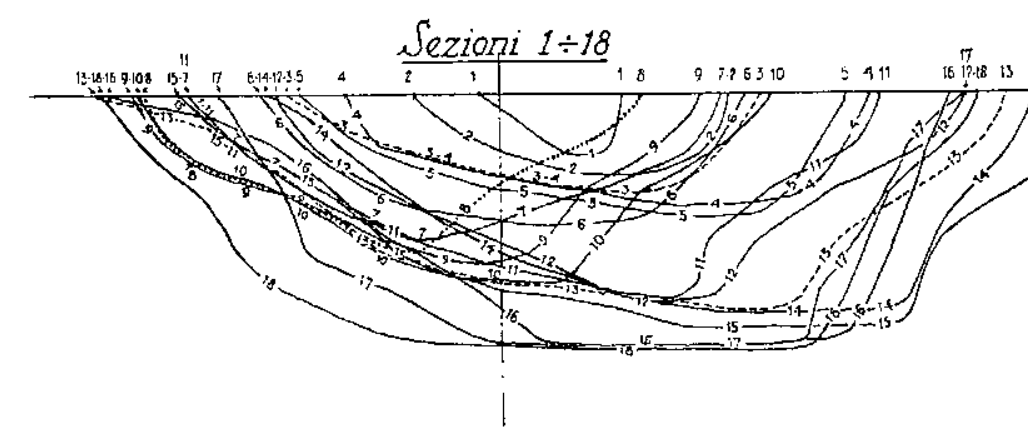

\section{Lago di SAURis \\ Invaso quota $\mathrm{m} .980$ Sezioni verticali Scale $\left\{\begin{array}{l}\text { Ascisse }: 1 \mathrm{~cm}=20 \mathrm{~m} \\ \text { Ordinate }: 1 \mathrm{~cm}=10 \mathrm{~m}\end{array}\right.$}
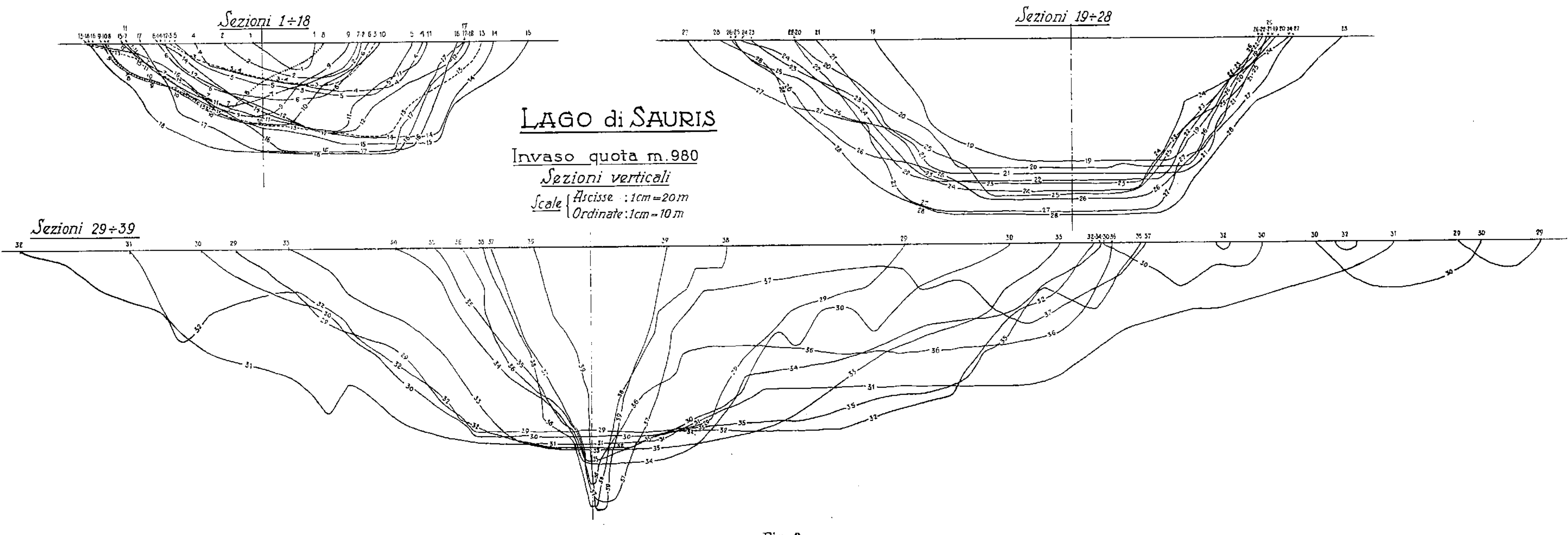
Hidaka integrando Ia [?] con le condizioni [3], ha provato che questo equivale alla ricerca del valore minimo dell'integrale:

$$
I(u)=\int_{0}^{1}\left\{\left(\frac{d u}{d z}\right)^{2}-\frac{\lambda}{\sigma(z)} u^{2}\right\} d z .
$$

Posto

$$
u=\sum_{0}^{m} A_{i} z(1-z) z^{i} \quad \text { con } A_{i}=\text { cost. }
$$

e sostituendo la [6] nella [4], determiniamo le $m+1$ costanti $\boldsymbol{A}_{0}, \boldsymbol{A}_{1}, \ldots$ $A_{m}$ in modo che la [5] diventi un minimo, il che presuppone l'annullamento dei rapporti delle derivate $\frac{\partial I}{\partial A_{i}}$.

Con opportuni calcoli si ottiene un sistema di $m+1$ equazioni:

$$
\left\{\begin{array}{l}
\left.\left(\frac{1}{3}-I_{0} \lambda\right) A_{0}+\frac{1}{6}-I_{1} \lambda\right) A_{1}+\left(\frac{1}{10}-I_{2} \lambda\right) A_{2}+\ldots \ldots=0 \\
\left(\frac{1}{6}-I_{1} \lambda\right) A_{0}+\left(\frac{2}{10}-I_{2} \lambda\right) A_{1}+\left(\frac{1}{10}-I_{3} \lambda\right) A_{2}+\ldots \ldots=0 \\
\left(\frac{1}{10}-I_{2} \lambda\right) A_{0}+\left(\frac{1}{10}-I_{3} \lambda\right) A_{1}+\left(\frac{3}{35}-I_{4} \lambda\right) A_{2}+\ldots=0
\end{array}\right.
$$

Eliminando lo $A_{i}$ costanti, si annulla il determinante dei coefficienti di $A_{0}, A_{1}, A_{2} \ldots \ldots$, la cui soluzione dà l'equazione dei periodi. In questa equazione si ha:

$$
I_{n}=\int_{0}^{1} \frac{z^{2}}{-(1-z)^{2} z^{n}} \frac{(z)}{\sigma(z)} d z
$$

Poiché la soluzione diviene tanto più difficoltosa quanto maggiore è il valore di $m$, Hidaki fornisee le soluzioni per $m=1$ ed $m=2$, soluzioni più che sufficienti per il nostro problema.

Per $m-1$, dalla [6] risulta $u=z(1-z)\left(A_{0}+A_{1} z\right)$, l'equazione del periodo della sessa uninodule si ottiene annullando il determinante formato dai coefficienti di $A_{6}$ e $A_{1}$ nelle prime due equazioni della [7]:

$$
\left.\begin{array}{ll:}
\left(\frac{1}{3}-I_{0} \lambda\right) & \left(\frac{1}{6}-I_{1} \lambda\right) \\
\left(\frac{1}{6}-I_{1} \lambda\right) & \left(\frac{2}{15}-I_{2} \lambda\right)
\end{array}\right)=0,
$$


sviluppando il qual' si ha:

$$
\left(I_{0} I_{2}-I_{1}^{2}\right) \lambda^{2}-\left(\frac{2}{1.5} I_{0}-\frac{1}{3} I_{1} \div \frac{1}{3} I_{2}\right) \lambda-\frac{1}{60}=0
$$

Sostituendo ad $I_{0}, I_{1}, I_{2}$ i valori numerici ricalvati dalla Tabella 1 , si ha un'equazione di $2^{\circ}$ in $\lambda$ le rui radici sono:

$$
{ }_{1} \lambda_{1}=0,00952 \quad, \quad \lambda_{2}=0,06171
$$

Ricordando la [4], dove $T_{1}=\frac{2 \pi a}{\sqrt{g_{1} \lambda_{1}}}$ e $T_{2}=\frac{2 \pi a}{\sqrt{g_{1} \lambda_{2}}}$, siamo finalmente arrivati alla determinazionc dei periodi della sessa uni e binodale:

$$
T_{1}=6^{m} 03 \mathrm{~s} \quad T_{2}=2^{\mathrm{m}} 2 \cdot 2 \times
$$

Per $m=2$, sempere dalla [6]: si ha

$$
u=z(1-z)\left(A_{0}+A_{1} z \cdot A_{2} z^{2}\right) .
$$

L'equazione dei poriodi si ottieno sviluppando e annullando il determinante formato dai coefieienti di $A_{0}, A_{1}, A_{2}$, nelle prime tre equazioni del sistema [7]:

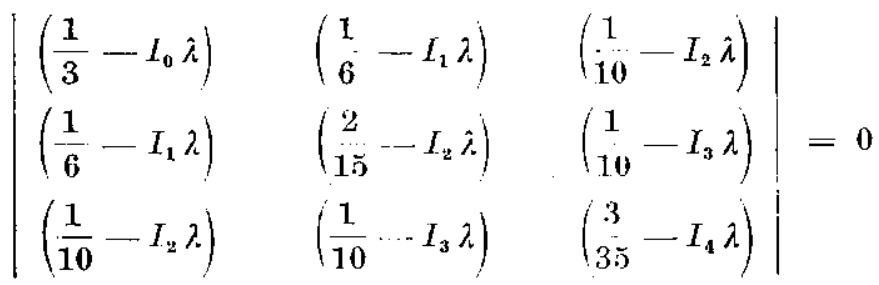

$$
\begin{aligned}
& \left(I_{0} I_{2} I_{4}-I_{0} I_{3}^{2}-I_{1}^{2} I_{4}+2 I_{1} I_{2} I_{3}-I_{2}^{3}\right) \lambda^{3}-\left[\frac{3}{35}\left(I_{0} I_{2}-I_{1}^{2}\right)+\right. \\
& +\frac{1}{5}\left(I_{1} I_{2}+I_{1} I_{3}-I_{0} I_{3}\right)+\frac{2}{15} I_{0} I_{4}+ \\
& \left.+\frac{1}{3}\left(I_{2} I_{3}+I_{2} I_{4}-I_{1} I_{4}-I_{3}^{2}\right)\right] \hat{\lambda}^{2}+ \\
& +\left(\frac{1}{700} I_{0}-\frac{3}{350} I_{1}+\frac{53}{2100} I_{2}-\frac{1}{30} I_{3}+\frac{1}{60} I_{4}\right) \lambda-\frac{1}{10500}=0 \text {. }
\end{aligned}
$$




\begin{tabular}{|c|c|c|c|c|c|c|c|c|c|c|c|c|c|}
\hline Sezioni & $\begin{array}{c}\Delta x \\
10^{2} \mathrm{~m}\end{array}$ & $\begin{array}{c}b(x) \\
10^{2} \mathrm{~m}\end{array}$ & $\begin{array}{l}S(x) \\
10^{3} \mathrm{~m}^{2}\end{array}$ & $\begin{array}{c}v(x) \\
10^{4} \mathrm{~m}^{2}\end{array}$ & $\begin{array}{r}V(x) \\
10^{4} \mathrm{~m}^{2}\end{array}$ & $\begin{array}{c}\sigma(x)= \\
=S(x) \cdot b(x) \\
10^{5} \mathrm{~m}^{3}\end{array}$ & $z=\frac{V}{a}$ & $\Delta z$ & $\mid \begin{array}{c}M= \\
\frac{z^{2}(1-z)^{2}}{\sigma(z)} \Delta z \\
10^{-10} \mathrm{~m}^{-3}\end{array}$ & $\begin{array}{c}M z z \\
10^{-10} \mathrm{~m}^{-3}\end{array}$ & $\begin{array}{c}M_{z^{2}} \\
10^{-10} \mathrm{~m}^{-3}\end{array}$ & $\begin{array}{c}W / \tilde{z}^{3} \\
10^{-10} \mathrm{~m}^{-3}\end{array}$ & $\begin{array}{c}M z^{4} \\
10^{-10} \mathrm{~m}^{-3}\end{array}$ \\
\hline 0 & 0 & 0 & 0 & 0 & 0 & 0 & 0 & 0 & 0 & 0 & 0 & 0 & 0 \\
\hline 1 & 1 & 0,58 & 0,494 & 0,4116 & 0,4116 & 0,2865 & 0.00233 & 0.00233 & 0.00439 & 0.00001 & 0 & 0 & 0 \\
\hline 2 & 2 & 1,29 & 1,579 & $1,21.88$ & 1,6304 & 2,0369 & 0.00924 & 0.00691 & 0.02850 & 0.00026 & 0.000003 & 0 & 0 \\
\hline 3 & 3 & 1,93 & 2,590 & 1,4552 & 3,0856 & 4,9987 & 0.01748 & 0.00824 & 0.04863 & 0.00085 & 0.000015 & 0 & 0 \\
\hline 4 & 4 & 2,14 & 3,600 & 1,1948 & 4,2804 & 7,7040 & 0.02425 & 0.00677 & 0.04921 & 0.00119 & 0.00003 & 0.000001 & 0 \\
\hline 5 & 5 & 2,24 & 3,951 & 1,6220 & 5,9024 & 8,8502 & 0.03344 & 0.00919 & 0.10851 & 0.00363 & 0.00012 & 0.000004 & 0 \\
\hline 6 & 6 & 1,98 & 3,889 & 2,1968 & 8,0992 & 7,7002 & 0.04588 & 0.01244 & 0.30954 & 0.01420 & 0.00065 & 0.000030 & 0.000001 \\
\hline 7 & 7 & 2,06 & 4,189 & 2,0700 & 10,1692 & 8,6293 & 0.05761 & 0.01173 & 0.40073 & 0.02309 & 0.00133 & 0.000077 & 0.000004 \\
\hline 8 & 8 & 2,04 & 3,731 & 2,1702 & 12,3394 & 7,6112 & 0.06991 & 0.01230 & 0.68326 & 0.04777 & 0.00334 & 0.000233 & 0.000016 \\
\hline 9 & 9 & 2,34 & 4,970 & 2,1068 & 14,4462 & 11,6298 & 0.08184 & 0.01193 & 0.57917 & 0.04740 & 0.00388 & 0.000318 & 0.000026 \\
\hline 10 & 10 & 2,60 & 6,1245 & 2,3746 & 16,8208 & 15,9237 & 0.09530 & 0.01346 & 0.62830 & 0.05988 & 0.00570 & 0.000543 & 0.000052 \\
\hline 11 & 11 & 2,81 & 7,3475 & 2,7040 & 19,5248 & 20,6465 & 0.11061 & 0.01531 & 0.71765 & 0.07938 & 0.00878 & $0.00097 \mathrm{I}$ & 0.000107 \\
\hline 12 & 12 & 2,79 & 7,144 & 2,8152 & 22,3400 & 19,9318 & 0.12656 & 0.01595 & 0.97788 & 0.12376 & 0.01567 & 0.001983 & 0.000251 \\
\hline 13 & 13 & 3,70 & 10,174 & 3,3008 & 25,6408 & 37,6438 & 0.14526 & 0.01870 & 0.76581 & 0.11124 & 0.01616 & 0.002347 & 0.000341 \\
\hline 14 & 14 & 3,14 & 9,9005 & 3,2406 & 28,8814 & 31,0876 & 0.16362 & 0.01836 & 1.10600 & 0.18096 & 0.02961 & 0.004845 & 0.000793 \\
\hline 15 & 15 & 3,82 & 12,188 & 3,4400 & 32,3214 & 46,5582 & 0.18311 & 0.01949 & 0.93661 & 0.17150 & 0.03140 & 0.005750 & 0.001053 \\
\hline 16 & 16 & 3,44 & 11,098 & 3,6336 & 35,9550 & 38,1771 & 0.20370 & 0.02059 & 1.41903 & 0.28906 & 0.05888 & 0.011994 & 0.002443 \\
\hline 17 & 17 & 3,06 & $11, \tilde{5} 40$ & 3,0140 & 38,9690 & 35,3124 & 0.22077 & 0.01707 & 1.43062 & 0.31584 & 0.06973 & 0.015394 & 0.003399 \\
\hline 18 & 18 & 3,56 & 14,038 & 3,2580 & 42,2270 & 49,9753 & 0.23923 & 0.01846 & 1.22354 & 0.29271 & 0.07002 & 0.016751 & 0.004007 \\
\hline 19 & 19 & 3,77 & 16,880 & 3,9504 & 46,1774 & 63,6376 & 0.26161 & 0.02238 & 1.77721 & 0.46494 & 0.12163 & 0.031820 & 0.008324 \\
\hline 20 & 20 & 4,57 & 20,204 & 4,1708 & 50,3482 & 92,3323 & 0.28524 & 0,02363 & 1.06377 & 0.30343 & 0.08655 & 0.024688 & 0.007042 \\
\hline 21 & 21 & 4,30 & 21,684 & 4,4256 & 54,7738 & 93,2412 & 0.31031 & 0.02507 & 1.23152 & 0.38215 & 0.11858 & 0.036797 & $0.011+18$ \\
\hline 22 & 22 & 4,46 & 22,845 & 4,3920 & 59,1658 & 101,8887 & 0.33519 & 0.02488 & 1.21254 & 0.40643 & 0.13623 & 0.045663 & 0.015306 \\
\hline 23 & 23 & 3,84 & 24,262 & $4,61.04$ & 63,7762 & 93,1661 & 0.36131 & 0.02612 & 1.48459 & 0.53640 & 0.19380 & 0.070022 & 0.025300 \\
\hline 24 & 24 & 5,26 & 23,110 & 5,0448 & 68,8210 & 121,5586 & 0.38989 & 0.02858 & 1.33036 & 0.51869 & 0.20223 & $0.0788+7$ & 0.030742 \\
\hline 25 & 25 & 5,08 & 24,116 & 5,0974 & 73,9184 & 122,5093 & 0.41877 & 0.02888 & I.39662 & 0.58486 & 0.24493 & 0.102569 & 0.042953 \\
\hline 26 & 26 & 5,02 & 27,182 & 5,1072 & 79,0256 & 136,4536 & 0.44771 & 0.02894 & 1.29668 & 0.58054 & 0.25991 & 0.116364 & 0.052097 \\
\hline 27 & 27 & 5,80 & 31,322 & 5,7994 & 84,8250 & 181,6676 & 0.48056 & 0.03285 & 1.12676 & 0.54148 & 0.26021 & 0.125047 & 0.060093 \\
\hline 28 & 28 & 5,97 & 33,627 & 6,0688 & 90,8938 & 200,7532 & 0.51494 & 0.03438 & 1.06843 & 0.55018 & 0.28330 & 0.145882 & 0.075120 \\
\hline 29 & 29 & 7,20 & 34,639 & 6,6464 & 97,5402 & 249,4008 & 0.55260 & 0.03766 & 0.92300 & 0.51005 & 0.28186 & 0.155756 & 0.086071 \\
\hline 30 & 30 & 10,79 & 43,489 & 8,3460 & 105,8862 & 469,2463 & 0.59988 & 0.04728 & 0.58049 & 0.34822 & 0.20890 & 0.125315 & 0.075174 \\
\hline 31 & 31 & 12,02 & 72,418 & 11,8180 & 117,7042 & 870,4644 & 0.66683 & 0.06695 & 0.37962 & 0.25314 & 0.16880 & 0.112561 & 0.075059 \\
\hline 32 & 32 & 12,52 & 57,4925 & 11,5948 & 129,2990 & 719,8061 & 0.73252 & 0.06569 & 0.35038 & 0.25666 & 0.18801 & 0.137721 & 0.100883 \\
\hline 33 & 33 & 7,32 & 43,220 & 8,6968 & 137,9958 & 316,3704 & 0.78179 & 0.04927 & 0.45327 & 0.35436 & 0.27704 & 0.216587 & 0.169326 \\
\hline 34 & 34 & 6,72 & 35,739 & 6,9064 & 144,9022 & 240,1661 & 0.82092 & 0.03913 & 0.35212 & 0.28906 & 0.23730 & 0.194804 & 0.159918 \\
\hline 35 & 35 & 6,72 & 41,437 & 6,0654 & 150,9676 & 278,4566 & 0.85528 & 0.03436 & 0.18902 & 0.16166 & 0.13827 & 0.118260 & 0.101145 \\
\hline 36 & 36 & 6,20 & 31,8665 & 6,5688 & 157,5364 & 197,5723 & 0.89250 & 0.03722 & 0.17347 & 0.15482 & 0.13818 & 0.123326 & 0.110068 \\
\hline 37 & 37 & 6,24 & 20,983 & 11,2454 & 168,7818 & 130,9339 & 0.95620 & 0.06370 & 0.08538 & 0.08164 & 0.07806 & 0.074641 & 0.071372 \\
\hline 38 & 38 & 3,73 & 12,378 & 4,9428 & 173,7246 & 46,1699 & 0.98421 & 0.02801 & 0.01468 & 0.01445 & 0.01422 & 0.013995 & 0.013774 \\
\hline 39 & 39 & 1,26 & 6,876 & 1,8552 & 175,5798 & 8,6638 & 0.99472 & 0.01051 & 0.00360 & 0.00358 & 0.00356 & 0.003541 & 0.003522 \\
\hline \multirow[t]{3}{*}{40} & \multirow[t]{3}{*}{39,7} & \multirow[t]{3}{*}{0} & \multirow[t]{3}{*}{0} & \multirow[t]{3}{*}{0,9324} & \multirow[t]{3}{*}{176,5122} & \multirow[t]{3}{*}{0} & \multirow[t]{3}{*}{1.00000} & \multirow[t]{3}{*}{0.00528} & 0 & 0 & 0 & 0 & 0 \\
\hline & & & & & & & & & 27.91089 & 9.05947 & 3.95689 & 2.11545 & 1.30720 \\
\hline & & & & & & & & & $\mathrm{I}_{0}$ & & $\mathrm{I}_{2}$ & $\mathrm{I}_{3}$ & $\mathrm{I}_{4}$ \\
\hline
\end{tabular}


Dalla Tabella $\mathrm{I}$, sostituiti ad $I_{0}, I_{1}, I_{2}, I_{3}, I_{4}$ i loro valori numerici

$$
\begin{aligned}
& I_{0}=27,91089 ; \quad I_{1}=9,0594 \pi ; \quad I_{2}=3,95690 ; \\
& I_{3}=2,115 \pm 4 ; \quad I_{4}=1,30720 \text { ， }
\end{aligned}
$$

abbiams, un'equazione di $3^{\circ}$ in 2 :

$$
188972 \lambda^{3}-34583 \lambda^{2}+1337 \lambda-9,52381=0 \text {. }
$$

Mediante lapplicazione del metodo di Newton per successive approssimazioni otteniamo la prima radice corletta di $\lambda\left({ }_{2} \lambda_{1}=0,00920\right)$ : dividendo poi la [12] per la radice trovata si ha m'equazione di $2^{\circ}$ in $\lambda$, risolvendo la quale abbianng i valori $\mathrm{di}_{2} \lambda_{2}$ e ${ }_{2} \lambda_{3}$ :

$$
{ }_{2} \hat{\lambda}_{1}=0,00920, \quad \quad{ }_{2} \lambda_{2}=0,04134, \quad{ }_{2} \hat{\lambda}_{33}=0,13247 .
$$

Sostituendo nella [f] rispettivamente le tre ladici trovate, si ricavano i periodi corrispondenti alla sessa uni-bi e tri-nodale:

$$
T_{1}=6^{\mathrm{m}} 09^{\mathrm{s}} \quad T_{2}=2 \mathrm{~m} 54^{\mathrm{s}} \quad T_{3}=1^{\mathrm{m}} 37^{\mathrm{s}} .
$$

5. - Nodi. Per la determinazione dei nodi ri rifacciamo alla [6] lirnitandoci al caso $m=2$.

Per $m=2$,

$$
u=A_{0} z(1-z)\left(1+\frac{A_{1}}{A_{0}} z+\frac{A_{2}}{A_{0}} z^{2}\right),
$$

da cui derivando lispetto a $\approx$ e licordando che nei nodi

$$
\frac{d u}{d z}=0
$$

otteniano

$$
\frac{d u}{d z}=4 \frac{-A_{2}}{A_{0}} z^{3}+3\left(\begin{array}{l}
A_{1} \\
A_{0}
\end{array}-\frac{A_{2}}{A_{0}}\right) z^{2}+2\left(1-\frac{A_{1}}{A_{0}}\right) z-1=0 .
$$

Per risolvere la [15], ci serviamo ancora una volta della [7], ricavando - da una qualunque coppia di equazioni del sistema - i, valori numerici dei rapporti $\frac{A_{1}}{A_{0}} \frac{A_{2}}{A_{0}}$;

$$
\begin{array}{ll}
\frac{A_{1}}{A_{0}}=-1,56949 & A_{2} \\
A_{0} & =+0,85246 .
\end{array}
$$

Sostituiti questi valori nella [15], otteniamo un'equazione di $3^{\circ}$ in $z$, che risolveremo ricorrendo al metodo di Newton e rammentando la condizione ai limiti $0<z<1$ :

$$
3,40984 z^{3}-7,26585 z^{2}+5,13898 \approx-1=0 .
$$


Risolvendo la [16], vediamo che l'unica radice possibile, delle tre ottenute, è

$$
{ }_{2} \tilde{\nu}_{1}=0,31223,
$$

la quale rappresenta il valore dell'uninodo.

L'uninodo cade quindi fra le sezioni 21 e 22 a 'a $\mathrm{km} 2,14$ dall'estremo 0 del lagro (v. Tab. I, Fig. 1).

Sostituendo nella [15] ai rapporti $\frac{A_{1}}{A_{0}}$ e $\frac{A_{2}}{A_{0}}$ i valori numerici ottenuti e servendoci del valore della radice ${ }_{2} \lambda_{2}$, dalla [7] si ha:

$$
16,25872 z^{3}-27,76689 z^{2}+12,38190 z-1=0 .
$$

Le radici utili, comprese cioè fra 0 ed 1 sono:

$$
{ }_{2} z_{1}=0,10320, \quad{ }_{2} z_{2}=0,58381 .
$$

I due nodi, quindi, cadono rispettivamente fra le sezioni 10 e 11 ad una distanza di $\mathrm{km} \mathrm{1,05}$ dall'estremo 0 del lago, e fra le sezioni 29 e 30 a circa $1 \mathrm{~km}$ dalla diga (Tab. I e Fig. 1).

6. - Ampiezze. Per determinare le ampiezze, la formula

$$
\eta=-\frac{d u}{d z} a \quad(a=\text { superficie totale del lago })
$$

in particolare per il caso $m=2$, si puó scrivere:

$$
\zeta=\frac{\eta}{a A_{0}}=-\left\{4-\frac{A_{2}}{A_{0}} z^{3}+3\left(\frac{A_{1}}{A_{0}}-\frac{A_{2}}{A_{0}}\right) z^{2}+2\left(1-\frac{A_{1}}{A_{0}}\right) z-1\right\} .
$$

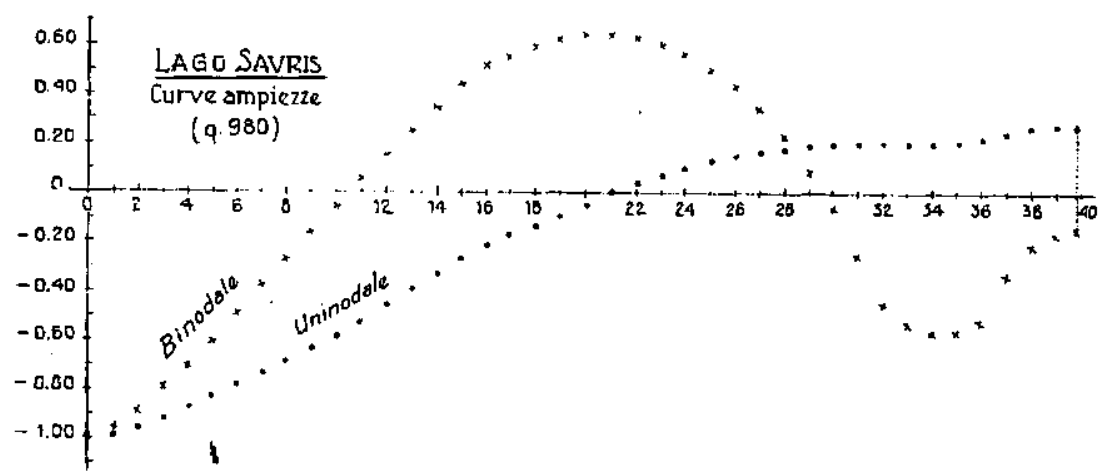

Fì‥t 
'T a b e l I a I I

\begin{tabular}{|c|c|c|c|}
\hline Sezioni & $\begin{array}{c}\Delta x \\
10^{2} \mathrm{n} 1\end{array}$ & $\zeta^{\prime}$ & $\zeta^{\prime \prime}$ \\
\hline 0 & 0 & -1 & $\ldots 1$ \\
\hline 1 & 1 & $\ldots, 98807$ & $-0,97130$ \\
\hline 2 & 2 & $-0,95317$ & $-0,88807$ \\
\hline 3 & 3 & $-0,91840$ & $-0,79209$ \\
\hline 4 & 4 & $-0,87962$ & $-0,71589$ \\
\hline 5 & 5 & $-0,83618$ & $-0,61645$ \\
\hline 6 & 6 & $-0,77915$ & $\ldots, 48867$ \\
\hline 7 & 7 & $-0,72741$ & $\cdots-0,37576$ \\
\hline 8 & 8 & $--0,67509$ & $-0,26460$ \\
\hline 9 & 9 & $-0,62624$ & $\ldots-0,16380$ \\
\hline 10 & 10 & $-0,57328$ & $\ldots, 05807$ \\
\hline II & 11 & $-0,51583$ & $-0,05197$ \\
\hline 12 & 12 & -- 0,45910 & $+0,15518$ \\
\hline 13 & 13 & $\cdots-0,39637$ & $+\quad 0,26254$ \\
\hline 14 & 14 & $-0,33874$ & $\div 0,35382$ \\
\hline 15 & 15 & $-0,28168$ & $+0,43606$ \\
\hline 16 & 16 & $-0,22583$ & $+0,50756$ \\
\hline 17 & 17 & $--0,18292$ & + 0,55513 \\
\hline 18 & 18 & $--0,13974$ & $\div 0,59562$ \\
\hline 19 & 19 & $-0,09181$ & $-0,62997$ \\
\hline 20 & 20 & $-0,04618$ & +. 0,65002 \\
\hline 21 & 21 & $\cdots, 00306$ & †. 0,65437 \\
\hline 22 & 22 & - $\quad 0,03462$ & $+0,64297$ \\
\hline 23 & 23 & $+0,06911$ & $+0,61585$ \\
\hline 24 & 24 & $+0,10125$ & $-0,57035$ \\
\hline 25 & 25 & f- 0,12826 & |. 0,50973 \\
\hline 26 & 26 & $+0,15040$ & $+0,43694$ \\
\hline 27 & 27 & $+0,17004$ & $+0,34217$ \\
\hline 28 & 28 & $+0,18524$ & $+0,23325$ \\
\hline 29 & 29 & $+0,19643$ & $+0,10667$ \\
\hline 30 & 30 & ; 0,20417 & $-0,05472$ \\
\hline 31 & 31 & $\div 0,20706$ & $-0,26929$ \\
\hline 32 & 32 & $+0,20591$ & $\ldots 0,43875$ \\
\hline 33 & 33 & $+0,20603$ & $-0,52217$ \\
\hline 34 & 34 & $+0,20857$ & $-0,55308$ \\
\hline 35 & 35 & $+0,21362$ & $-0,54943$ \\
\hline 36 & 36 & $+0,22301$ & $-0,50833$ \\
\hline 37 & 37 & $\div 0,25171$ & $-0,33369$ \\
\hline 38 & 38 & + 0,27049 & $-0,20990$ \\
\hline 39 & 39 & $+0,27863$ & $-0,15540$ \\
\hline 40 & 39,7 & - 0,28297 & $-0,12627$ \\
\hline
\end{tabular}


Riprendiamo i valori ${ }_{2} \dot{\lambda}_{1}{ }^{2}{ }_{2} \lambda_{22}$, corrispondenti alla sessia uni e binodale, rispettivamente, lat [19] divin'me:

$$
\begin{aligned}
& \zeta^{-1}=3,4098+z^{3}-7,26585 z^{2}-5,13898 z-1 \\
& \because 1=16,25872 z^{3}-27,76689 z^{4}+12,38190 z-1 .
\end{aligned}
$$

Servendeci ora doi valuri numerici di z dati dalla Tabella I e sostituendoli mella [20], si ha l'andimento dolle ampiezze per le due sesse considerate. Isa Fig. $f$ dà uma rappresentazione grafical di detto andamento, mentre i risultati del adcolo sono contenuti nolla Tabella II.

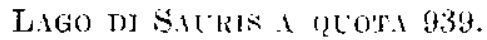

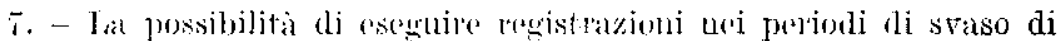

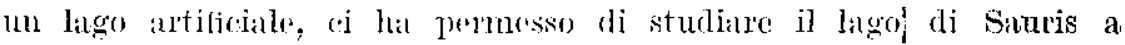
q. 939 (quola di alleggerinstento della diga), applieando lo stesso metodo usato pel il liggo ad invaso pieno, allo seopo di determinarne $i$ periodi, i uodi " le ampiezze della sessa mij- e binodale.

Va premesso che la diminuzione del tivello del hacino, e quindi

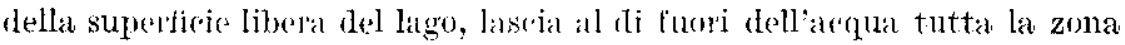
compresil precedentemente fra le sezimi $0 \ldots 13$ (Fig. 1). La sezione 0 (lel lago a quota 939, roincide pertanto com la seyione 13 ded lago a quota 9 so.

Il gyafieo in Fig. 5 rapporesenta la culva nomale, cuello in Fig. 6 l'andamento delle sezioni rertirali alla quota in esame.

I dati che camatterizzano la forma del lago sono contenuti nella Trabella III, dalla quale si tagggono anche i valori muneriei prre l'applicazione del metodo di ITidaka:

$$
\begin{gathered}
I_{0}=80,50657 \quad I_{1}=28,62169 \quad I_{2}=13,60640 \quad I_{3}=7,81607 \\
I_{4}=5,12866,
\end{gathered}
$$

valori necessari al alcolo dei perjorli, dei nodi e delle ampiezze.

8. - Periodi. Trascurando il caso $m=0$, per $m=1$ l'cquazione di $2^{\circ}$ grado in $\lambda$ risulta:

$$
276,203456 \lambda^{2}-5,729090 \lambda+0,016667=0,
$$

le cui radici sono:

$$
{ }_{1} \lambda_{1}=0,00350 \quad{ }_{1} \lambda_{3}=0,01.724 .
$$


T a b o l l a I I I

\begin{tabular}{|c|c|c|c|c|c|c|c|c|c|c|c|c|c|}
\hline Sezioni & $\begin{array}{c}\Delta x \\
10^{2} \mathrm{~m}\end{array}$ & $\begin{array}{c}b(x) \\
10^{2} \mathrm{~m}\end{array}$ & $\begin{array}{c}S(x) \\
10^{3} \mathrm{~m}^{2}\end{array}$ & $\begin{array}{c}v(x) \\
10^{4} \mathrm{~m}^{2}\end{array}$ & $\begin{array}{c}V(x) \\
10^{4} \mathrm{~m}^{2}\end{array}$ & $\begin{aligned} & \sigma(x)= \\
&= S(x) \cdot b(x) \\
& 10^{5} \mathrm{~m}^{3}\end{aligned}$ & $z=\frac{V}{a}$ & $\Delta z$ & $\mid \begin{array}{c}M= \\
\frac{z^{2}(1-z)^{2}}{\sigma(z)} \Delta z \\
10^{-10} \mathrm{~m}^{-3}\end{array}$ & $\begin{array}{c}M z \\
10^{-10} \mathrm{~m}^{-3}\end{array}$ & $\begin{array}{c}M z^{2} \\
10^{-10} \mathrm{~m}^{3}\end{array}$ & $\begin{array}{c}M z^{3} \\
10^{-10} \mathrm{~m}^{-3}\end{array}$ & $\begin{array}{c}M z^{4} \\
10^{-10} \mathrm{~m}^{-3}\end{array}$ \\
\hline $0=13$ & 0 & 0 & 0 & 0 & 0 & 0 & 0 & 0 & 0 & 0 & 0 & 0 & 0 \\
\hline $1=14$ & 1 & 1,11 & 0,4385 & 0,760 & 0,760 & 0,487 & 0,00882 & 0,00882 & 0,14465 & 0,00128 & 0,00001 & 0 & 0 \\
\hline $2=15$ & 2 & 1,50 & 0,833 & 1,320 & 2,080 & 1,2495 & 0,02415 & 0,01533 & 0,67452 & 0,01629 & 0,00039 & 0,00001 & 0 \\
\hline $3=16$ & 3 & 1,50 & 1,395 & 1,690 & 3,770 & 2,0925 & 0,04377 & 0,01962 & 1,65004 & 0,07222 & 0,00317 & 0,00014 & 0,00001 \\
\hline $4=17$ & 4 & 1,68 & 1,467 & 1,540 & 5,310 & 2,465 & 0,06165 & 0,01788 & 2,42989 & 0,14980 & 0,00923 & 0,00057 & 0,00003 \\
\hline $5=18$ & 5 & 2,20 & 2,079 & 1,970 & 7,280 & 4,574 & 0,08452 & 0,02287 & 2,98911 & 0,25264 & 0,02134 & 0,00180 & 0,00015 \\
\hline $6=19$ & 6 & 2,64 & 3,537 & 2,320 & 9,600 & 9,338 & 0,11146 & 0,02694 & 2,83139 & $0,3 \mathrm{I} 559$ & 0,03517 & 0,00392 & 0,00044 \\
\hline $7=20$ & 7 & 2,83 & 4,986 & 2,720 & 12,320 & 14,110 & 0,14304 & 0,03158 & 3,36327 & 0,48108 & 0,06881 & 0,00984 & 0,00141 \\
\hline $8=21$ & 8 & 3,10 & 6,366 & 2,840 & 15,160 & 19,735 & 0,17601 & 0,03297 & 3,51460 & 0,61860 & 0,10888 & 0,01916 & 0,00337 \\
\hline $9=22$ & 9 & 3,14 & 7,169 & 3,181 & 18,341 & 22,511 & 0,21294 & 0,03693 & 4,60886 & 0,98141 & 0,20897 & 0,04450 & 0,00947 \\
\hline $10=23$ & 10 & 3,11 & 6,476 & 3,172 & 21,513 & 20,140 & 0,24977 & 0,03683 & 6,41947 & 1,60339 & 0,40045 & 0,10002 & 0,02498 \\
\hline $11=24$ & 11 & 2,89 & 7,303 & 2,841 & 24,354 & 21,106 & 0,28275 & 0,03298 & 6,42780 & 1,81746 & 0,51390 & 0,14531 & 0,04109 \\
\hline $12=25$ & 12 & 2,92 & 6,708 & 2,750 & 27,104 & 19,587 & 0,31468 & 0,03193 & 7,58337 & 2,38633 & 0,75091 & 0,23630 & 0,07436 \\
\hline $13=26$ & 13 & 3,66 & 9,242 & 3,330 & 30,434 & 33,826 & 0,35334 & 0,03866 & 5,96524 & 2,10776 & 0,74476 & 0,26315 & 0,09298 \\
\hline $14=27$ & 14 & 3,40 & 11,562 & 3,481 & 33,915 & 39,311 & 0,39375 & 0,04041 & 5,85541 & 2,30557 & 0,90782 & 0,35746 & 0,14075 \\
\hline $15=28$ & 15 & 4,00 & 13,479 & 4,180 & 38,095 & 53,916 & 0,44228 & 0,04853 & 5,47418 & 2,42112 & 1,07080 & 0,47359 & 0,20946 \\
\hline $16=29$ & 16 & 3,90 & 12,825 & 3,905 & 42,000 & 50,0175 & 0,48762 & 0,04534 & 5,65843 & 2,75916 & 1,34540 & 0,65604 & 0,31990 \\
\hline $17=30$ & 17 & 4,33 & 14,654 & 4,129 & 46,129 & 63,452 & 0,53556 & 0,04794 & 4,67415 & 2,50329 & 1,34064 & 0,71799 & 0,38452 \\
\hline $18=31$ & 18 & 9,86 & 27,981 & 7,062 & 53,191 & 275,893 & 0,61755 & 0,08199 & 1,65620 & 1,02279 & 0,63162 & 0,39006 & 0,24088 \\
\hline $19=32$ & 19 & 6,20 & 23,326 & 7,979 & 61,170 & 144,621 & 0,71018 & 0,09263 & 2,71406 & 1,92747 & 1,36886 & 0,97214 & 0,69040 \\
\hline $20=33$ & 20 & 4,78 & 17,893 & 5,520 & 66,690 & 85,5285 & 0,77427 & 0,06409 & 2,28801 & 1,77154 & 1,37164 & 1,06202 & 0,82228 \\
\hline $21=34$ & 21 & 4,10 & 12,791 & 4,476 & 71,166 & 52,443 & 0,82624 & 0,05197 & 2,04242 & 1,68753 & 1,39430 & 1,15202 & 0,95185 \\
\hline $22=35$ & 22 & 4,86 & 17,324 & 4,646 & 75,812 & 84,195 & 0,88018 & 0,05394 & 0,71201 & 0,62670 & 0,55161 & 0,48551 & 0,42734 \\
\hline $23=36$ & 23 & 5,32 & 8,459 & 5,163 & 80,975 & 45,002 & 0,94012 & 0,05994 & 0,41958 & 0,39446 & 0,37084 & 0,34863 & 0,32776 \\
\hline $24=37$ & 24 & 1,40 & 5,983 & 2,353 & 83,328 & 8,376 & 0,96744 & 0,02732 & 0,32238 & 0,31188 & 0,30173 & 0,29190 & 0,28240 \\
\hline $25=38$ & 25 & 1,14 & 4,755 & 1,481 & 84,809 & 5,421 & 0,98463 & 0,01719 & 0,07220 & 0,07109 & 0,07000 & 0,06892 & 0,06786 \\
\hline $26=39$ & 26 & 0,68 & 2,724 & 0,825 & 85,634 & 1,852 & 0,99421 & 0,00958 & 0,01533 & 0,01524 & 0,01515 & 0,01507 & 0,01497 \\
\hline \multirow[t]{3}{*}{$27=40$} & \multirow[t]{3}{*}{26,70} & \multirow[t]{3}{*}{0} & \multirow[t]{3}{*}{0} & \multirow[t]{3}{*}{0,500} & \multirow[t]{3}{*}{86,134} & \multirow[t]{3}{*}{0} & \multirow[t]{3}{*}{1} & \multirow[t]{3}{*}{0,00579} & 0 & 0 & 0 & 0 & 0 \\
\hline & & & & & & & & & 80,50657 & 28,62169 & 13,60640 & 7,81607 & 5,12866 \\
\hline & & & & & & & & & $\mathrm{I}_{0}$ & $I_{1}$ & $\mathrm{I}_{2}$ & $\mathrm{I}_{3}$ & $\mathrm{I}_{4}$ \\
\hline
\end{tabular}




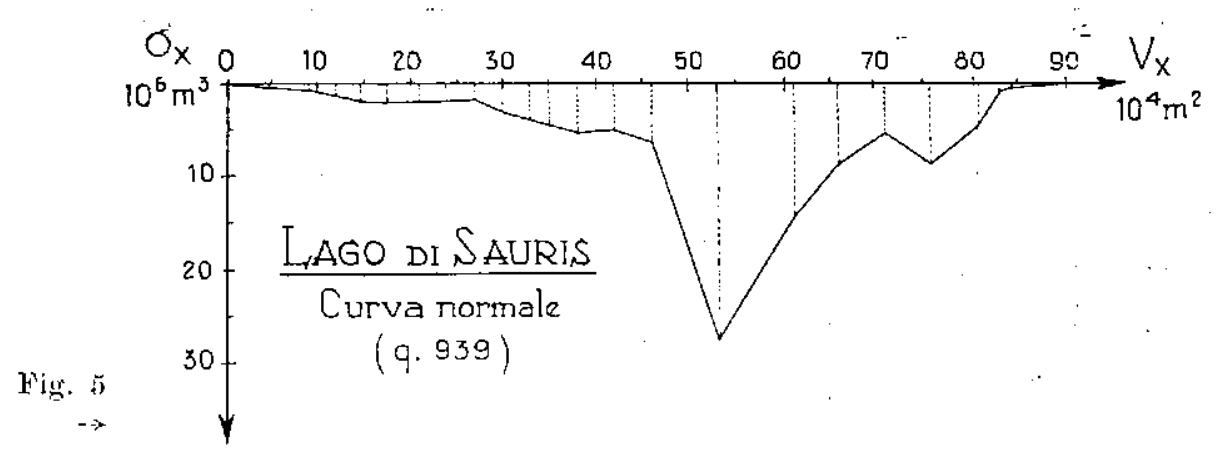

Sezioniviticath $3 \div 39$

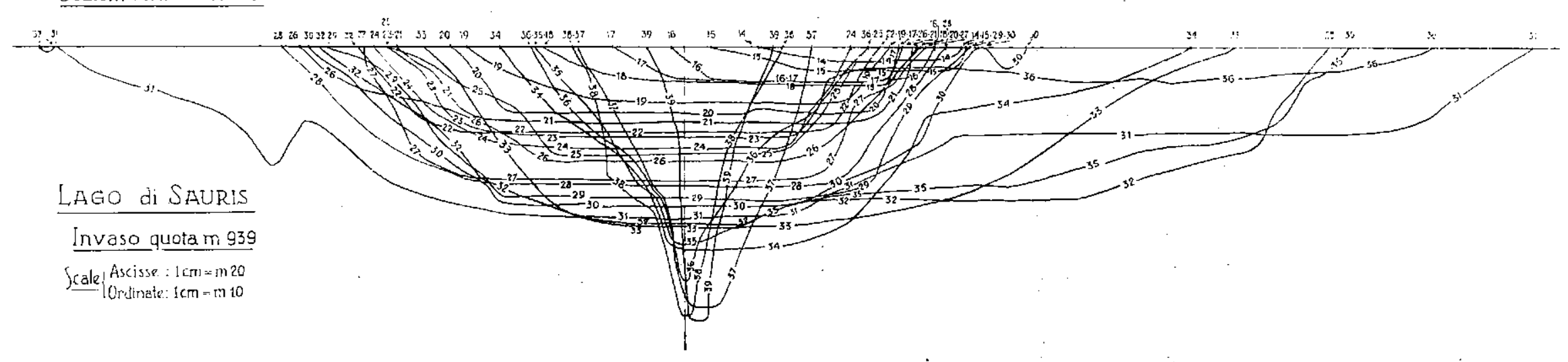

risr. 6 
Sostituendo neIla [4], i valori della [22], si hanno i periodi corrispondenti rispettivamente alla s'ssat uni- a bi-nodale:

$$
T_{1}=4 \mathrm{~m} 5 \mathrm{2} \mathrm{s} \quad T_{\mathrm{z}--} \cdot 2 \mathrm{~m} 12 \mathrm{~s}
$$

Per $m$ :- 2 , si ha la seguente equatzione di $3^{\circ}$ grado in $\hat{A}$ :

$67,073756 \lambda^{3}-3,212071 \lambda^{2}+0,038071 \lambda-0,0000952381=0$,

risolvendo la quale si hanno le tre radici:

$$
{ }_{2} \hat{\lambda}_{1}=0,003416 . \quad{ }_{2} \gamma_{2}=0,013361 . \quad{ }_{2} \hat{\lambda}_{3}=0,031111 .
$$

Introdotti i valori della [24] nella [4], si ottengono i valori dei periodi per la sessa uni- bi- e trinodale del lago:

$$
\begin{array}{ll}
T_{1}=4^{\mathrm{m}} 56^{\mathrm{s}} & \text { (uninodale) } \\
T_{2}=2^{\mathrm{m}} 29^{\mathrm{s}} & \text { (binodale) } \\
T_{3}=1^{\mathrm{m}} 38^{\mathrm{s}} & \text { (trinodale) }
\end{array}
$$

9. - Nordi. Nel caso $m-2$, sostituendo nell'equazione dei nodi [15] il valore ${ }_{2} \hat{\lambda}_{1}=0,003416$, si ha un'equazione di $3^{0}$ grado in $z$ :

$$
3,02960 z^{3}--6,5765+2 z^{2}+4.86956 z-1=0 .
$$

L'unica madiece possibile $(0<z<1)$ è quella minore dell'unità:

$$
{ }_{2} z_{1}=0,33021 \text {. }
$$

che rappresenta il valore "orrispondente all'minodo; questo viene dunque a trovarsi fra le sezioni $25-24$ a dista $1,24 \mathrm{~km}$ cira dall'estremo 0 del lago (v. Fig. 1, Tabella III).

Sostituendo poi nella $\left[15 \mid\right.$ la radice $i_{2}=0,013361$, si ha:

$$
14,11492 z^{3}-24.92157 z^{2}+1.596692-1-0
$$

le rui soluzioni valide sono:

$$
{ }_{2} z_{1}=0,11175 \quad{ }_{2} z_{2}-0,60362 .
$$

I due binodi adono, pertanto, fra le sezioni 19-20 e 30-31 e distano rispettivamente eirea $0,6 \mathrm{~km}$ dallestremo 0 del lago, e circa $0,9 \mathrm{~km}$ dalla diga (V. Fig. 1. Tabella III). 
10. - Ampiezze. Per $m=2$, le equazioni delle ampiezze relative alle oseillazioni dell'uninodale e della binodale sono [6]; [i]]:

$$
\begin{aligned}
& \zeta^{\prime}=3,02960 z^{3}-6,57654 z^{2}+4,86956 z-1 \\
& \zeta^{\prime \prime}=14,11492 z^{3}-24,92155 z^{2}+11,55691 z-1 .
\end{aligned}
$$

Sostituendo nella [30] i valori numerici di $z$ dati dalla Tab. III, si ottengono le ampiezze delle oscillazioni in corrispondenza delle varie

\begin{tabular}{|c|c|c|c|}
\hline Sezioni & $\begin{array}{c}\Delta x \\
10^{2} \mathrm{~m}\end{array}$ & $\zeta^{\prime}$ & $\zeta^{\prime \prime}$ \\
\hline & & & \\
\hline $0=13$ & 0 & -1 & -1 \\
\hline $1=14$ & 1 & $-0,95758$ & $-0,90005$ \\
\hline $2=15$ & 2 & $-0,88617$ & $-0,73515$ \\
\hline $3=16$ & 3 & $-0,79925$ & $-0,54087$ \\
\hline $4=17$ & 4 & $-0,72408$ & $-0,37897$ \\
\hline $5=18$ & 5 & $-0,63356$ & $-0,19268$ \\
\hline $6=19$ & 6 & $-0,53474$ & $-0,00192$ \\
\hline $7=20$ & 7 & $-0,42914$ & $+0,18456$ \\
\hline $8=21$ & 8 & $-0,33014$ & $+0,33899$ \\
\hline $9=22$ & 9 & $-0,23202$ & $+0,46720$ \\
\hline $10:=23$ & 10 & $-0,14677$ & $+0,55187$ \\
\hline $11=24$ & 11 & $-0,08042$ & $+0,59438$ \\
\hline $12=25$ & 12 & $-0,02446$ & $+0,60882$ \\
\hline $13=26$ & 13 & $-0,03317$ & $\begin{array}{r}0,59467\end{array}$ \\
\hline $14=27$ & 14 & $+0,08269$ & $+0,54842$ \\
\hline $15=28$ & 15 & $\div 0,12936$ & $+0,45756$ \\
\hline $16=29$ & 16 & $\div 0,16204$ & $+0,34627$ \\
\hline $17=30$ & 17 & $+0,18704$ & $+0,20961$ \\
\hline $18=3 I$ & 18 & $+0,21264$ & $-0,04301$ \\
\hline $19=32$ & 19 & $+0,22649$ & $-0,30613$ \\
\hline $20=33$ & 20 & $+0,23403$ & $-0,44033$ \\
\hline $21=34$ & 21 & $+0,24267$ & $-0,50290$ \\
\hline $22=35$ & 22 & $+0,25696$ & $-0,51025$ \\
\hline $23=38$ & 23 & $+0,28275$ & $-0,43331$ \\
\hline $24=37$ & 24 & $+0,29897$ & $-0,36042$ \\
\hline $25=38$ & 25 & $+0,31081$ & $-0,30807$ \\
\hline $26==39$ & 26 & $+0,31807$ & $-0,27256$ \\
\hline $27=40$ & 26,7 & $+0,32262$ & $-0,24973$ \\
\hline
\end{tabular}

T a bella IV 
sezioni rlel lago; i valori ottenuti - riportati nella Tabella IV - i banno permesso di costruire graficamente le curve delle ampiezze (Fig. 7).

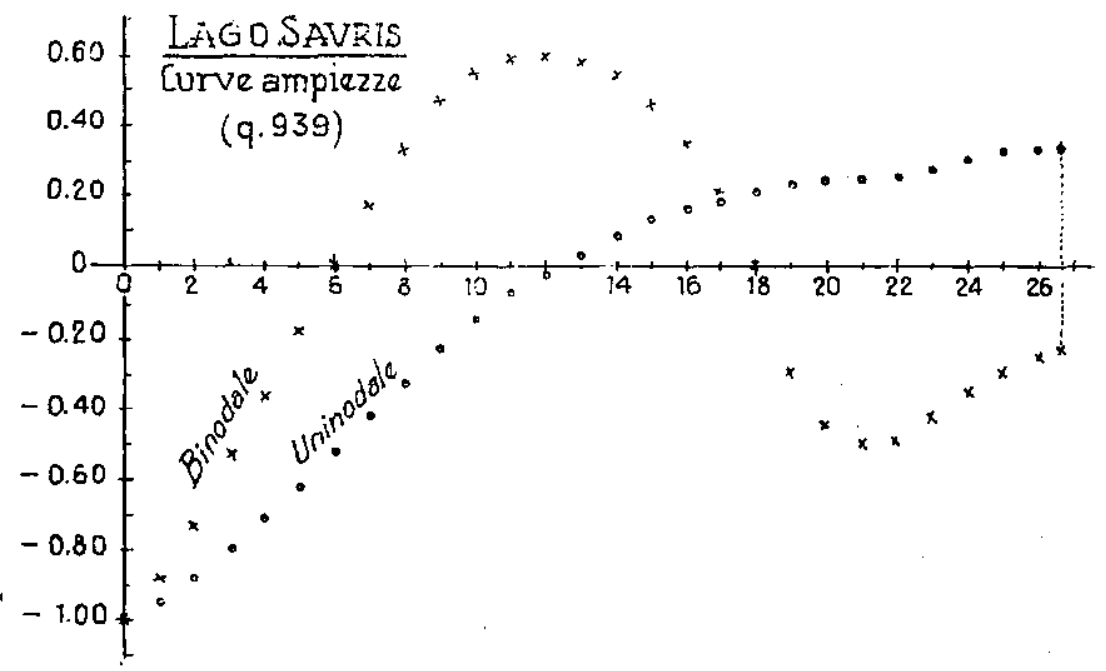

Fig. 7

\section{Conclusiont.}

In questa prima parte del lavoro sulle oscillazioni libere di un lago artificiale, quale e il lağo di Sauris, ei siamo limitati a determinare $i$ periodi della sessa uni e bi-nodale nei casi $m=1$ ed $m=2$, la posizione dei nodi e l'andamento delle ampiczze, alle 'quote 980 (invaso pieno) e 939 (quota alleggerimento diga).

Dai valori numerici si sono potuti ricavare i grafici relativi.

Ia seconda parte, che è in fase di compimento, sarè dedicata al confronto fra i dati ottenuti per via teorica e quelli ottenuti per via sperimentale.

Ringraziamo il Prof. P. Caloi per l'assistenza e per $i$ preziosi consigli fornitici, $i$ quali ai hanns permesso di portare a termine questa prima parte dello studio del lago di Sauris. 


\section{BIBLIOGRAFIA}

(1) Chrystax, G., On the hydrodynamical Theory of Seiehes. 'Transactions of the Royal Society of lidinburg. XLI (1905), XI」V (1906), XIJVI (1907-8).

(2) НIDAKA K., 1 pplication of Ritz's larialion to the Determination of Seiches in a Lake. The Memoirs of the Imperial Harine Observatory, VI, 2, (1960).

(3) Calor P., Le sesse del tago di Garda. Parte I, "Annali di Geofisica ", I, 1, (1948).

(4) C'alor P., Le sesse del Lago di Garda. Parte II, "Aunali di Geofisica", I, 2, (1948). 